\title{
Host serum factor triggers germination of Clostridium perfringens spores lacking the cortex hydrolysis machinery
}

\begin{abstract}
Correspondence
Mahfuzur R. Sarker

sarkerm@oregonstate.edu
\end{abstract}

Received 17 February 2011

Accepted 26 July 2011

\author{
Daniel Paredes-Sabja ${ }^{1} \dagger$ and Mahfuzur R. Sarker ${ }^{1,2}$ \\ 1Department of Biomedical Sciences, College of Veterinary Medicine, Oregon State University, \\ Corvallis, OR 97331, USA \\ 2Department of Microbiology, College of Science, Oregon State University, Corvallis, OR 97331, \\ USA
}

\begin{abstract}
Clostridium perfringens type $A$ is the causative agent of a variety of histotoxic and enteric diseases. The ability of $C$. perfringens spores to germinate in vivo might be due to the presence of nutrient germinants in the host tissue and blood. In the current study, we investigated the ability of spores of $C$. perfringens wild-type and mutation strains to germinate in blood. Results indicate that spores of all three surveyed C. perfringens wild-type isolates germinated better in blood than in brain heart infusion (BHI) broth. However, as expected, spores lacking germinant receptor (GR) protein GerAA or GerKB germinated like wild-type spores in BHI broth and blood. Strikingly, while spores lacking GR proteins GerKA and GerKC showed significantly decreased germination in $\mathrm{BHI}$ broth, these spores germinated well in blood, suggesting that blood factor(s) can trigger spore germination through a GR-independent pathway. Using C. perfringens spores lacking cortex lytic enzymes ( $\Delta \operatorname{cspB}$ or $\Delta s / e C \Delta s / e M)$, we were able to identify a host serum germination factor with peptidoglycan hydrolysing activity that (i) restored the colony-forming efficiencies of $\Delta c s p B$ and $\Delta s / e C \Delta s / e M$ spores up to $\sim 5-20 \%$ of that of total colony-forming spores;

(ii) increased the number of c.f.u. of decoated $\Delta c s p B$ and $\Delta s / e C \Delta s / e M$ spores to $\sim 99 \%$ of that of colony-forming spores; (iii) and finally lost enzymic activity after heat inactivation, consistent with serum germination factor being an enzyme. Further characterization demonstrated that serum germination factor is very likely lysozyme, which can form a stable high molecular mass complex of $\sim 120 \mathrm{kDa}$ in serum. In conclusion, the current study indicates that a host serum germination factor with peptidoglycan hydrolysing activity is capable of triggering germination of C. perfringens spores by directly degrading the spore peptidoglycan cortex. Collectively, this study contributes to our understanding of the mechanism of in vivo germination of spores of C. perfringens.
\end{abstract}

\section{INTRODUCTION}

Clostridium perfringens is a Gram-positive, spore forming, anaerobic bacterium, which is highly associated with a wide array of gastrointestinal (GI) and histotoxic diseases in both humans and animals (Amimoto et al., 2007; Keyburn et al., 2008; McClane, 2007). However, the most common cause of $C$. perfringens-associated food poisoning in humans is the consumption of $C$. perfringens vegetative cells followed by sporulation in the gut (McClane, 2007;

†Present address: Departmento de Ciencias Biológicas, Facultad de Ciencias Biológicas, Universidad Andrés Bello, Santiago, Chile.

Abbreviations: Ca-DPA, calcium dipicolinate; Gl, gastrointestinal; GR, germinant receptor; HMM, high molecular mass; HMMC, high molecular mass complex; HRP, horseradish peroxidase; HS, human serum; LMM, low molecular mass.
Paredes-Sabja \& Sarker, 2009). The early steps in the development of $C$. perfringens-associated gas gangrene and other non-food-borne GI illnesses, such as antibioticassociated diarrhoea, occur when $C$. perfringens spores ubiquitously found in the environment come in contact with the host, where spores undergo germination followed by outgrowth, cell proliferation and toxin secretion. Therefore, C. perfringens spore germination can be considered as the most essential and earliest step in the progression of gas gangrene and antibiotic-associated diarrhoea.

Bacterial spores break their dormancy when they sense the presence of nutrients (termed germinants) in the environment, the germination process being triggered by germinants binding to their cognate germinant receptors (GRs) (Paredes-Sabja et al., 2011; Setlow, 2003). In C. perfringens, the main GR involved in sensing nutrient 
germinants is GerKA-KC (Paredes-Sabja et al., 2008b, 2009a). Binding of the germinant to its cognate receptor leads to a variety of biophysical events, including the release of monovalent cations followed by the release of the spore's large deposit of calcium dipicolinate (Ca-DPA) (Setlow, 2003). The release of dipicolinic acid chelated at a $1: 1$ ratio with monovalent cations, predominantly $\mathrm{Ca}^{2+}$ (Ca-DPA), in Bacillus subtilis activates one of the two redundant cortexlytic enzymes (Paidhungat et al., 2001). However, in $C$. perfringens, Ca-DPA triggers spore germination through the GerKA-KC receptor (Paredes-Sabja et al., 2008b). Spore peptidoglycan cortex hydrolysis in C. perfringens is triggered through a Ca-DPA-independent pathway (Paredes-Sabja et al., 2009b, c). Degradation of the spore peptidoglycan cortex eliminates a physical constraint, allowing the spore core to uptake water to levels found in growing bacteria, and consequently to the resumption of metabolism and outgrowth (Setlow, 2003).

Besides nutrient germinants, host-specific factors also play important roles in spore germination. Previous studies have shown that in vitro lysozyme can trigger germination of bacterial spores, bypassing the GRs and directly degrading the spore cortex-lytic enzyme (Lund \& Peck, 1994; Peck et al., 1992). Indeed, in a recent study with Bacillus anthracis (Giebel et al., 2009), it has been shown that spores lacking all cortex lytic enzymes are able to germinate in vitro with whole blood and serum despite their lack of germination in nutrient-rich media, and kill $25 \%$ of the challenged mice in 4 days, suggesting that hostspecific enzymes with peptidoglycan-hydrolysing activity might be involved in spore germination and virulence in vivo. Consequently, in this study we found that the main GR proteins, GerKA and GerKC, are not required for $C$. perfringens spore germination in blood due to the presence of a host serum germination factor with peptidoglycan hydrolysing activity (most likely lysozyme) that triggers significant germination by directly degrading the spore's peptidoglycan cortex.

\section{METHODS}

Bacterial strains. Our initial attempt in using spores of C. perfringens strain 13, an experimentally proven gas gangrene isolate (Awad et al., 2001), failed due to its inability to sporulate under laboratory conditions. Indeed, an early stop codon was detected in the spoOA gene, encoding a master regulator of sporulation, in strain 13 (Huang et al., 2004; Shimizu et al., 2002). Therefore, we surveyed spores from three other C. perfringens wild-type strains: ATCC 3624 (type A, enterotoxin-negative), F4969 (non-food-borne type A, enterotoxinpositive) (Sarker et al., 2000), and SM101 (electroporatable derivative of a food poisoning type A strain NCTC8798, enterotoxin-positive) (Zhao \& Melville, 1998). All these wild-type isolates are capable of producing alpha toxin and thus have the potential to cause gas gangrene. SM101 mutation strain derivatives used in this study were: (i) those lacking GR-encoding genes, such as DPS101 ( $\Delta$ gerKA-KC),

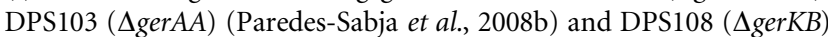
(Paredes-Sabja et al., 2009a); (ii) those lacking either the cortex lytic enzymes, DPS110 ( $\Delta$ sleC $\Delta$ sleM) (Paredes-Sabja et al., 2009c), or CspB, involved in processing of pro-SleC into mature SleC, DPS117 $(\Delta c s p B)$
(Paredes-Sabja et al., 2009b). Spores of strains DPS117 and DPS110 are defective in peptidoglycan cortex hydrolysis and thus also defective in colony formation on brain heart infusion (BHI) agar plates (ParedesSabja et al., 2009b, c).

Spore preparation and purification. C. perfringens isolates were grown overnight in fluid thioglycollate medium (Difco; BD) at $37{ }^{\circ} \mathrm{C}$ as described by Kokai-Kun et al. (1994). For C. perfringens sporulating cultures, $10 \mathrm{ml}$ Duncan Strong sporulating medium (Duncan \& Strong, 1968) was inoculated with $0.2 \mathrm{ml}$ fluid thioglycollate culture, and incubated overnight at $37{ }^{\circ} \mathrm{C}$ to form spores as confirmed by phase-contrast microscopy. Large amounts of spores were prepared by scaling-up the latter procedure. Spore preparations were cleaned by repeated washing with sterile distilled water and centrifugation until spores were $>99 \%$ free of sporulating cells, cell debris and germinated spores, before being resuspended in distilled water at a final $\mathrm{OD}_{600}$ of $\sim 6$ and stored at $-20{ }^{\circ} \mathrm{C}$ (Paredes-Sabja et al., 2008b).

Decoating of spores. Spore coats were extracted as previously described (Paredes-Sabja et al., 2008a). Briefly, spore suspensions at an $\mathrm{OD}_{600}$ of $\sim 20$ were incubated in $1 \mathrm{ml} 50 \mathrm{mM}$ Tris/HCl ( $\mathrm{pH} 8.0$ ), $8 \mathrm{M}$ urea, $1 \%(\mathrm{w} / \mathrm{v}) \mathrm{SDS}, 50 \mathrm{mM}$ DTT for $90 \mathrm{~min}$ at $37{ }^{\circ} \mathrm{C}$, and washed five times with $150 \mathrm{mM} \mathrm{NaCl}$ and twice with distilled water. Decoated spore suspensions were adjusted to an $\mathrm{OD}_{600}$ of $\sim 6$ and stored at $-20{ }^{\circ} \mathrm{C}$ until use.

Germination assays. Germination of spores of $C$. perfringens SM101 wild-type and $\mathrm{Ger}^{-}$mutation strains in BHI broth (Difco; BD) and sheep blood was quantified by loss of spore heat resistance. Briefly, the initial c.f.u. value was determined by plating serially diluted aliquots of heat-activated $\left(80{ }^{\circ} \mathrm{C}, 10 \mathrm{~min}\right)$ spore suspensions on $\mathrm{BHI}$ agar plates, incubating overnight under anaerobic conditions at $37{ }^{\circ} \mathrm{C}$ and counting the colonies. To determine the germinated spore fraction in BHI broth and sheep blood, heat-activated $\left(80^{\circ} \mathrm{C}\right.$, $10 \mathrm{~min}$ ) spores were resuspended in BHI broth and sheep blood to a final concentration of $10^{7}$ c.f.u. $\mathrm{ml}^{-1}$. After $60 \mathrm{~min}$ of incubation at $37{ }^{\circ} \mathrm{C}$, germinating spore suspensions were subjected to wet heat at $80{ }^{\circ} \mathrm{C}$ for $20 \mathrm{~min}$ to kill germinated spores, and aliquots were serially diluted and plated onto $\mathrm{BHI}$ agar, incubated overnight at $37^{\circ} \mathrm{C}$ under anaerobic conditions and the colonies counted. The germination percentage was calculated using the following formula: [1-(heat resistant c.f.u. $\left.\mathrm{ml}^{-1}\right) /\left(\right.$ initial c.f.u. $\left.\left.\mathrm{ml}^{-1}\right)\right] \times 100$.

To quantify the increase in c.f.u. counts of $C$. perfringens SM101 wildtype and $\mathrm{Ger}^{-}$mutation strain spores, aliquots of heat-activated spore suspensions at an $\mathrm{OD}_{600} \sim 1$ were directly plated onto $\mathrm{BHI}$ agar or incubated for $1 \mathrm{~h}$ at $37^{\circ} \mathrm{C}$ in sheep blood, and aliquots plated onto BHI agar and incubated anaerobically overnight at $37^{\circ} \mathrm{C}$. Results were expressed as c.f.u. $\mathrm{ml}^{-1} / \mathrm{OD}_{600}$ (c.f.u. $\mathrm{ml}^{-1}$ at $\mathrm{OD}_{600}=1$ ). To examine the germination of $C$. perfringens cortex lytic enzyme mutants, $50 \mu \mathrm{l}$ spore suspension with an $\mathrm{OD}_{600} \sim 6$ was added to $250 \mu \mathrm{BHI}$ broth, blood, serum or lysozyme, and incubated at $37{ }^{\circ} \mathrm{C}$ for $1-5 \mathrm{~h}$. For visualization, $5 \mu$ lgerminated $(5 \mathrm{~h}$ ) samples were applied to poly-L-lysine coated glass microscope slides and examined under phase-contrast microscopy. To quantify the germination, aliquots of $1 \mathrm{~h}$ germinated samples were serially diluted, plated onto $\mathrm{BHI}$ agar, incubated overnight at $37{ }^{\circ} \mathrm{C}$ under anaerobic conditions and the number of colonies counted. The colony-forming efficiency of untreated spores, expressed as c.f.u. $\mathrm{ml}^{-1} /$ $\mathrm{OD}_{600}$, was compared to those of decoated spores plated on BHI agar containing $1 \mu \mathrm{g}$ lysozyme $\mathrm{ml}^{-1}$.

Serum filtration and inactivation. Sheep serum was obtained by centrifugation $(3000 \mathrm{~g}, 10 \mathrm{~min}$ ) of defibrinated sheep blood (PML Microbiological). Sheep serum and human serum (HS) (BioWhittaker; Lonza) were fractionated into low molecular mass $(\mathrm{LMM})(<30 \mathrm{kDa})$ and high molecular mass $(\mathrm{HMM})(>30 \mathrm{kDa})$ fractions by centrifugation $(4000 \mathrm{~g}, 15 \mathrm{~min})$ using centrifugal filters 
with a nominal molecular mass limit of $30 \mathrm{kDa}$ (Amicon Ultra-4) and stored at $4{ }^{\circ} \mathrm{C}$ until use. Heat-inactivated serum was prepared by heating sheep serum and $\mathrm{HS}$ at $57{ }^{\circ} \mathrm{C}$ for $60 \mathrm{~min}$, which was cooled on ice and stored at $4{ }^{\circ} \mathrm{C}$ until use.

SDS-PAGE and Western blotting. Serum fractions were analysed with SDS-PAGE. Briefly, $1 \mu \mathrm{l}$ untreated sheep serum and HS, 10 and $5 \mu \mathrm{LMM}$ serum fraction and $1 \mu \mathrm{l}$ HMM serum fractions of sheep serum and HS were boiled with an equal volume of SDS-PAGE loading buffer (125 mM Tris-HCL, $20 \%$ glycerol, $4 \%$ SDS, $0.01 \%$ bromophenol blue, $2 \%$ 2-mercaptoethanol) and run on SDS-PAGE (18\% acrylamide) gels, and the gels were stained with Coomassie brilliant blue (Bio-Rad Laboratories).

For Western blotting, monoclonal anti-human lysozyme antibody (Abcam) and polyclonal anti-lysozyme antibody (Invitrogen) were used. Since the monoclonal anti-human lysozyme antibody epitope is disulfide bond dependent (Abcam), samples were mixed with SDS-PAGE loading buffer in the absence of $\beta$-mercaptoethanol and heat treatment. Proteins were run on SDS-PAGE (12\% acrylamide) gels and transferred to a PVDF (polyvinylidene fluoride) membrane (Millipore). These Western blots were probed with a 1:500 dilution of mouse monoclonal antihuman lysozyme antibody (Abcam) for $24 \mathrm{~h}$ at room temperature and then with 1:10000 dilution of goat anti-mouse IgG-horseradish peroxidase (HRP) conjugate (Promega) for $1 \mathrm{~h}$ at room temperature, in TBS ( $25 \mathrm{mM}$ Tris base, $\mathrm{pH} 7.4 / 150 \mathrm{mM} \mathrm{NaCl})$ with $4 \%$ BSA and $0.05 \%$ Tween. HRP activity was detected with a chemiluminescence detection system (Molecular Imager ChemiDoc XRS + System; BioRad) by using the PicoMax sensitive chemiluminescent HRP substrate (Rockland Immunochemicals). Each Western blot also included $10 \mu \mathrm{l}$ PageRuler multicolour broad range protein ladder (Fermentas).

\section{RESULTS}

\section{Blood triggers germination of spores of C. perfringens wild-type and GR mutation strain isolates}

Spores of all three surveyed C. perfringens wild-type strains, irrespective of their isolation source, germinated well in blood (Fig. 1). However, BHI-triggered spore germination varied between strains; the highest germination was observed with SM101, followed by F4969 and ATCC3624, as measured by $\sim 95,50$ and $25 \%$ germination, respectively (Fig. 1). When spores of SM101 derivatives with mutations in the GRencoding genes (i.e. $\triangle$ gerKA-KC, $\triangle$ gerKB and $\triangle$ gerAA spores) were assayed, the majority of $\triangle$ ger $A A$ and $\triangle$ gerKB spores germinated with either BHI broth or sheep blood (Fig. 1). Surprisingly, while only $\sim 50 \%$ of $\triangle$ gerKA-KC spores germinated in BHI broth, incubation of $\triangle g e r K A-K C$ spores in sheep blood significantly $(P<0.005)$ increased germination to $>90 \%$ (Fig. 1). These results indicate that at least GerKA$\mathrm{KC}$ is not required for sheep blood-triggered germination of C. perfringens spores, and suggest that an additional germination factor(s) present in the sheep blood might be triggering spore germination through a GR-independent pathway.

\section{Host serum germination factor possesses peptidoglycan hydrolysing activity}

To evaluate if factor(s) in sheep blood can trigger $C$. perfringens spore germination by directly degrading the

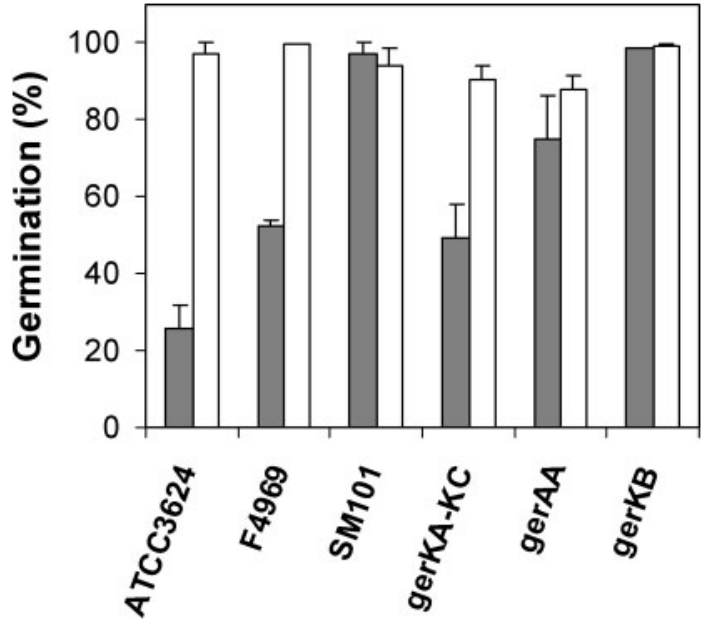

Fig. 1. Germination of $C$. perfringens spores in blood does not require GerKA-KC. Heat-activated spores of $C$. perfringens strains ATCC3624 (wild-type), SM101 (wild-type), F4969 (wild-type), DPS101 ( $\Delta$ gerKA-KC), DPS103 ( $\triangle$ gerAA) and DPS108 ( $\Delta$ gerKB) were incubated at $37{ }^{\circ} \mathrm{C}$ for $60 \mathrm{~min}$ in $\mathrm{BH}$ broth (grey bars) or sheep blood (white bars), and germination was measured by monitoring the loss of spore heat resistance as described in Methods.

spore peptidoglycan cortex, the germination of spores lacking cortex lytic enzymes were assayed. As expected, $\Delta \operatorname{csp} B$ and $\Delta$ sleC $\Delta$ sleM spores incubated in BHI broth prior to plating onto BHI agar resulted in a $\sim 100000$-fold lower c.f.u. value than seen when decoated $\Delta \operatorname{csp} B$ and $\Delta s l e C$ $\Delta$ sle $\mathrm{M}$ spores were directly plated onto $\mathrm{BHI}$ agar containing lysozyme (Fig. 2). Strikingly, when $\Delta \operatorname{csp} B$ and $\Delta$ sle $C \Delta$ sleM spores were incubated in sheep blood prior to plating onto BHI agar, a significant increase $(P<0.001)$ in c.f.u. counts was observed (Fig. 2). The $\Delta \operatorname{csp} B$ and $\Delta$ sle $C \Delta$ sleM spores yielded similar c.f.u. counts both in whole blood and serum (Fig. 2); however, c.f.u. counts of $\Delta \operatorname{csp} B$ spores incubated in HS were significantly higher than for those incubated in sheep serum and the opposite effect was found with $\Delta$ sleC $\Delta$ sleM spores (Fig. 2). Collectively, these results indicate that the germination factor is present in the serum of sheep and humans, and this serum germination factor possesses enzymic activity that can directly degrade the spore peptidoglycan cortex.

Spore coats are known to provide some protection from exogenous cortex hydrolysing enzymes (Klobutcher et al., 2006; Laaberki \& Dworkin, 2008). Therefore, if the serum germination factor possesses peptidoglycan hydrolysing activity it should increase the germination efficiency of $\Delta \operatorname{csp} B$ and $\Delta$ sle $C \Delta$ sleM spores upon removal of their coats. Some indirect evidence came from the observation that serum germination factor triggered germination in only $\sim 10 \%$ of total colony-forming $\triangle \operatorname{csp} B$ and $\Delta$ sleC $\Delta$ sleM spores (Fig. 2), suggesting that the serum germination factor was able to trigger germination presumably of coatdefective $\Delta \operatorname{csp} B$ and $\Delta$ sle $C \Delta$ sleM spores. We first compared 


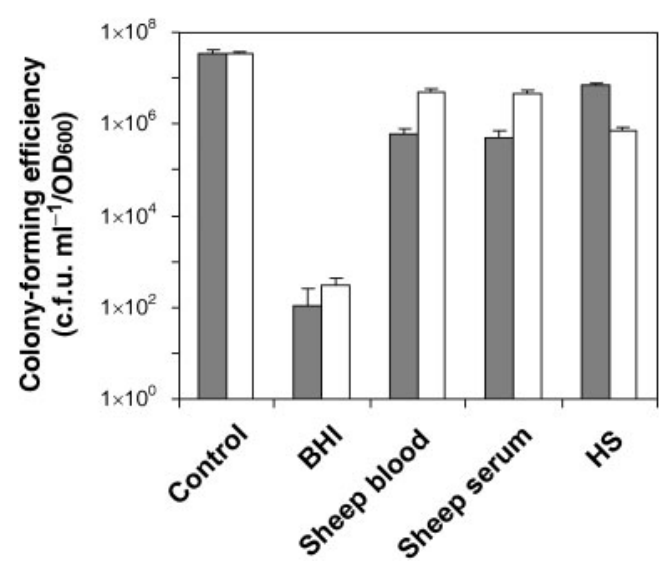

Fig. 2. Blood and serum increases colony-forming efficiency of $C$. perfringens spores lacking cortex lytic enzymes. Heat-activated spores of $C$. perfringens strains DPS117 $(\Delta c s p B)$ (grey bars) and DPS110 ( $\Delta$ sleC $\Delta$ sleM) (white bars) were incubated in $\mathrm{BHI}$ broth, blood serum at $37{ }^{\circ} \mathrm{C}$ for $60 \mathrm{~min}$, and colonies were counted after plating on $\mathrm{BHI}$ agar. As a control for the maximum colony-forming efficiency of spores of strains DPS117 and DPS110, decoated spores were heat activated, plated onto $\mathrm{BHI}$ agar containing lysozyme and the colonies counted.

the germination efficiency of decoated and untreated $\Delta c s p B$ spores under phase-contrast microscopy. As expected, similar fractions of untreated $\Delta \operatorname{csp} B$ spores became phase dark when incubated with HS and lysozyme for $5 \mathrm{~h}$ (Fig. 3). Decoating $\triangle c s p B$ spores increased phase dark spores to $>99 \%$ at $5 \mathrm{~h}$ incubation in either HS or lysozyme (Fig. 3), suggesting that $C$. perfringens spore coats provide some protection against the peptidoglycan cortex hydrolytic activity of the serum germination factor.

Next, germination experiments were repeated with untreated and decoated $\Delta c s p B$ and $\Delta$ sle $C \Delta s l e M$ spores, and the number of c.f.u. was counted. Untreated $\Delta \operatorname{csp} B$ and $\Delta$ sle $C \Delta s l e M$ spores incubated with lysozyme or HS yielded a higher number of c.f.u. than those directly plated onto BHI plates (Fig. 4). However, when decoated $\Delta \operatorname{csp} B$ and $\Delta$ sleC $\Delta$ sleM spores were germinated with HS, c.f.u. levels were restored to those of decoated spores plated directly onto BHI agar with lysozyme (Fig. 4). Similar results were obtained when these experiments were repeated with sheep serum (data not shown). Collectively, these results indicate that spore coats act as a physical barrier for serum factortriggered germination, and provide evidence that the serum germination factor has peptidoglycan hydrolysing activity that degrades the spore peptidoglycan cortex.

\section{Serum germination factor's peptidoglycan hydrolysing activity is heat labile and present in the HMM fraction of serum}

The heat stability of the serum germination factor was evaluated by heat inactivating $\left(57^{\circ} \mathrm{C}\right.$ for $\left.60 \mathrm{~min}\right)$ sheep serum and HS, and assaying for germination of $\triangle c s p B$ and $\Delta$ sle $C \Delta$ sle $M$ spores. The $\Delta \operatorname{csp} B$ and $\Delta$ sle $\Delta$ sleM spores incubated with heat-inactivated HS did not show an increase in their c.f.u. counts, which were similar to those with BHI broth (Fig. 5). Similar results were obtained with heat-inactivated sheep serum (data not shown). These results indicate that the serum germination factor's peptidoglycan hydrolysing activity is heat labile, which is (a) Un-BHI

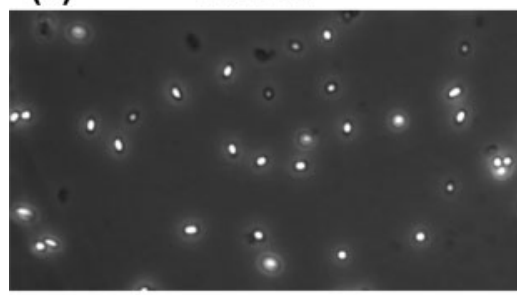

(d)

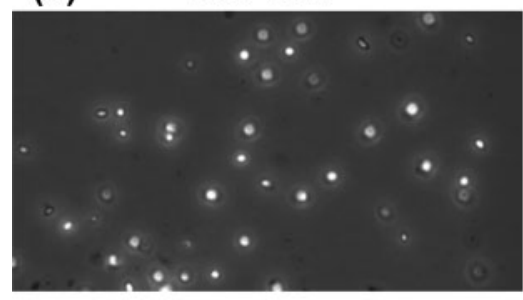

(b)

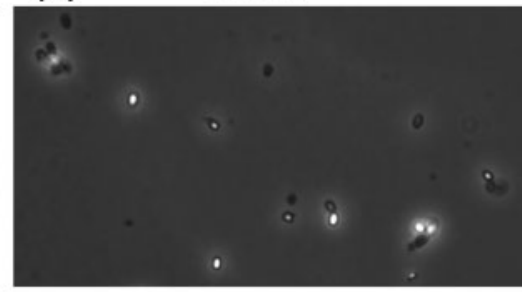

(e)

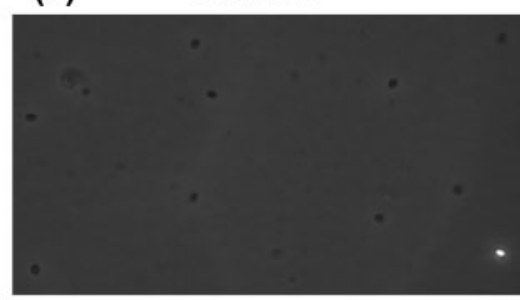

(c)

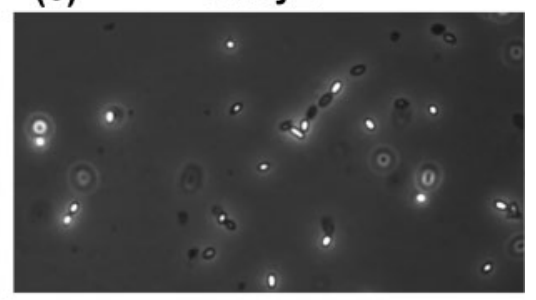

(f)

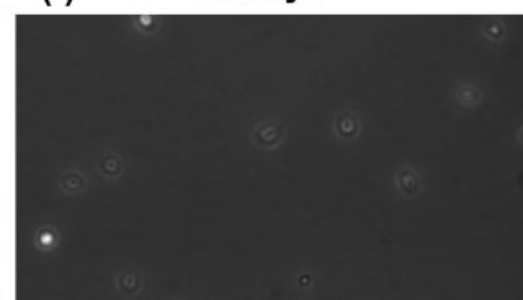

Fig. 3. HS increases germination of decoated $\Delta \operatorname{csp} B$ spores. Untreated $(a, b, c)$ and decoated (d, e, f) spores of strain DPS117 $(\Delta \operatorname{csp} B)$ were heat activated and incubated at $37{ }^{\circ} \mathrm{C}$ for $5 \mathrm{~h}$ in $\mathrm{BHI}$ broth $(\mathrm{a}, \mathrm{d}), \mathrm{HS}(\mathrm{b}, \mathrm{e})$ and $1 \mu \mathrm{g}$ lysozyme $\mathrm{ml}^{-1}$ (c, f), and then photographed under phase-contrast microscopy. 


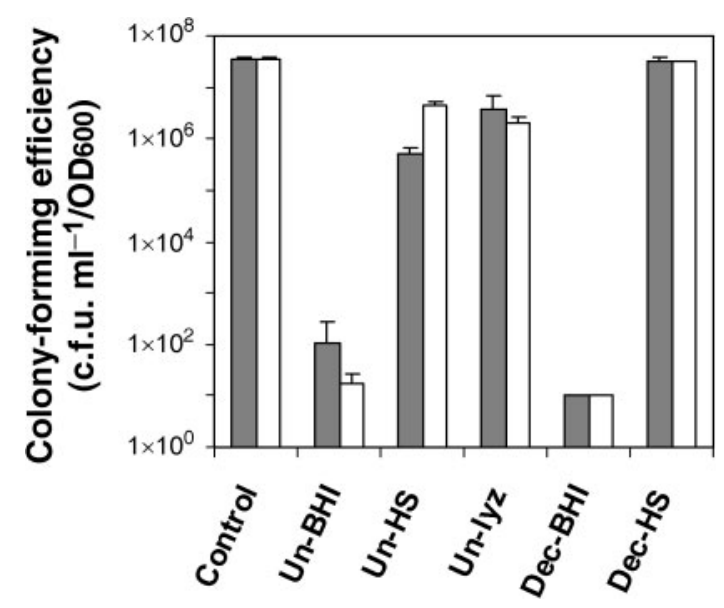

Fig. 4. HS increases colony-forming efficiency of decoated $\Delta c s p B$ and $\Delta$ sleC $\Delta$ sleM spores. Untreated or decoated spores of strains DPS117 $(\Delta \operatorname{csp} B)$ (grey bars) and DPS110 ( $\Delta$ sleC $\Delta$ s/eM) (white bars) were heat activated and incubated at $37{ }^{\circ} \mathrm{C}$ for $60 \mathrm{~min}$ with $\mathrm{BHI}$ or $\mathrm{HS}$, plated onto $\mathrm{BHI}$ agar and the colonies counted. Un$\mathrm{BHI}$, untreated spores in $\mathrm{BHI}$ broth; un-HS, untreated spores in $\mathrm{HS}$; dec-BHI, decoated spores in $\mathrm{BHI}$ broth; dec-HS, decoated spores in $\mathrm{HS}$; and control, decoated spores that were heat activated and plated onto $\mathrm{BHI}$ containing lysozyme.

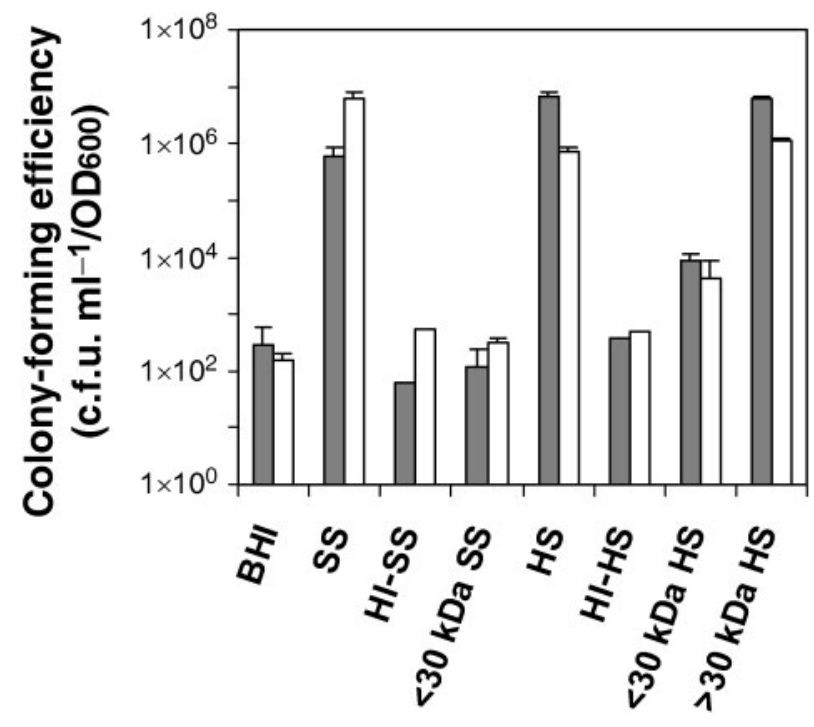

Fig. 5. Serum germination factor has a molecular mass $>30 \mathrm{kDa}$. Heat-activated spores of $C$. perfringens strains DPS117 $(\Delta \operatorname{csp} B)$ (grey bars) and DPS110 ( $\Delta$ s/eC $\Delta s / e M)$ (white bars) were incubated at $37{ }^{\circ} \mathrm{C}$ for $60 \mathrm{~min}$ with: $\mathrm{BHI}$ broth, sheep serum (SS), heat inactivated sheep serum (HI-SS), LMM sheep serum $(<30 \mathrm{kDa} S \mathrm{SS}), \mathrm{HS}$, heat inactivated HS (HI-HS), LMM HS $(<30 \mathrm{kDa} H S)$ or HMM HS $(>30 \mathrm{kDa} H S)$. The c.f.u. counts were determined by plating onto $\mathrm{BHI}$ agar. consistent with serum germination factor being an enzyme in nature (Fig. 4).

In an attempt to separate the protein with peptidoglycan hydrolysing activity, sheep serum and HS were fractionated by ultrafilter centrifugation with a filter with a molecular cut-off mass of $30 \mathrm{kDa}$. The filtered serum expected to contain species of a molecular mass $<30 \mathrm{kDa}$, hereafter LMM, was used for germination (Fig. 5). Sheep serumLMM was unable to induce germination of $\Delta c s p B$ and $\Delta s l e C$ $\Delta$ sleM spores; i.e. similar levels of c.f.u. were observed with sheep serum-LMM and BHI broth. Although slight increases in c.f.u. counts for $\Delta \operatorname{csp} B$ and $\Delta$ sleC $\Delta$ sleM spores were observed with HS-LMM, these were significantly $(P<0.05)$ lower than that with unfiltered HS (Fig. 5). When $\Delta \operatorname{csp} B$ and $\Delta s l e C \Delta s l e M$ spores were germinated in the serum fraction of a molecular mass of $>30 \mathrm{kDa}$ (HMM), similar c.f.u. levels as for unfiltered sheep serum and HS were observed (Fig. 5; data not shown). These results suggest that the serum germination factor's peptidoglycan hydrolysing activity is present in the HMM fraction of serum.

\section{Detection of lysozyme in the HMM fraction of serum}

In mammals, lysozyme is the only protein with peptidoglycan hydrolysing activity identified to date (Prager \& Jolles, 1996), and early studies have reported antimicrobial lysozyme activity with a molecular mass of $\sim 16 \mathrm{kDa}$ in $\mathrm{HS}$ (Inoue et al., 1959; Michael \& Braun, 1964). However, the serum is compromised by a 'network' of protein/peptide and protein-protein interactions, and removal of the highabundance serum carrier proteins also removes the lowabundance peptides and proteins (Zhou et al., 2004). This suggests that the serum germination factor, likely serum lysozyme, might be interacting with other serum proteins forming a HMM complex that cannot pass through the $30 \mathrm{kDa}$ filter membrane.

To investigate possible interactions of the serum germination factor (lysozyme) with HMM carrier proteins, HS, HMM and LMM were analysed by SDS-PAGE and Western blotting with monoclonal anti-human lysozyme antibody. While no LMM-specific protein band was detected on a SDS-PAGE gel, a smear of protein bands of $>30 \mathrm{kDa}$ was found in the HMM fraction (Fig. 6a). Strikingly, Western blotting under non-reducing conditions detected a $\sim 120$ $\mathrm{kDa}$ band immunoreactive to lysozyme $\mathrm{mAb}$ in $\mathrm{HS}$ and HMM (Fig. 6b). However, no immunoreactive band was detected in the LMM fraction of HS (Fig. 6b). These results were confirmed using a polyclonal anti-lysozyme antibody with essentially similar results (data not shown). Interestingly, a free lysozyme specific band with a predicted molecular mass of $\sim 16 \mathrm{kDa}$ was missing in the HMM fraction (Fig. 6b). Collectively, these results indicate that lysozyme is present in the serum forming a stable complex with HMM proteins, and is absent in the LMM fraction, suggesting that the serum germination factor with spore peptidoglycan hydrolysis activity might be serum lysozyme. 
(a)

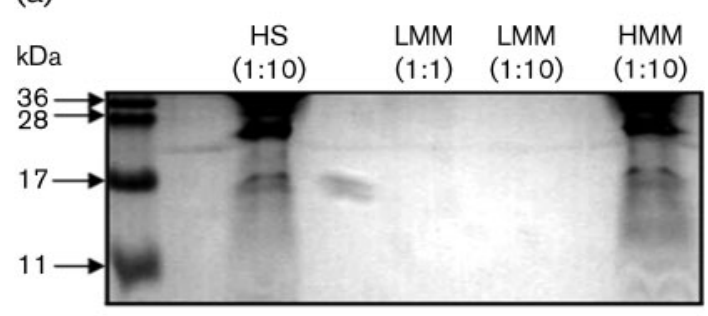

(b)

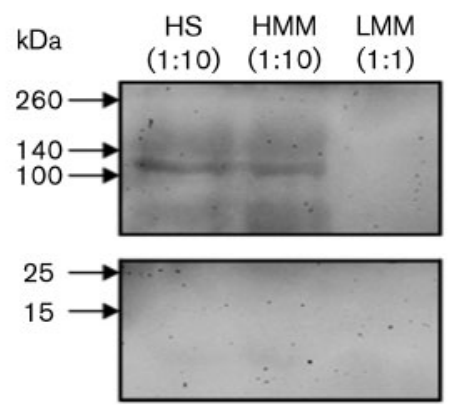

Fig. 6. Lysozyme forms a HMMC in HS. (a) HS was fractionated with a $30 \mathrm{kDa}$ cut-off molecular mass filter and run in a SDSPAGE gel. $\mathrm{HS}$ and the $>30 \mathrm{kDa}$ fraction (HMM) were mixed at a $1: 10$ ratio with SDS sample buffer, while the $<30 \mathrm{kDa}$ fraction (LMM) was mixed at a $1: 1$ or $1: 10$ ratio and loaded in an $18 \%$ SDS-PAGE gel. The gel was electrophoresed at $100 \mathrm{~V}$ for $1-2 \mathrm{~h}$ and stained with Coomassie brilliant blue. (b) Aliquots of HS, HMM and LMM fractions were separated under non-reducing conditions by SDS-PAGE, proteins transferred to a PVDF (polyvinylidene fluoride) membrane and probed with lysozyme mAb as described in Methods. The arrows indicate the relative locations of known molecular mass proteins.

\section{DISCUSSION}

The ability of $C$. perfringens spores to germinate, outgrow and return to vegetative cells with high speed (Paredes-Sabja et al., 2009a) seems to provide great advantage for this anaerobic bacterium in the development of gas gangrene and other non-food-borne GI illnesses, such as antibioticassociated diarrhoea. Despite the good understanding of the pathogenesis of $C$. perfringens-mediated gas gangrene and other non-food-borne illnesses, there is little knowledge available on the molecular basis involved in the early stages (i.e. in vivo spore germination and outgrowth) of these diseases. In this context, our present study offers several significant contributions. First, all surveyed C. perfringens spores germinated well in blood and the main GR proteins, GerKA and GerKC, are not required for this blood-triggered germination. It was surprising that while only $\sim 50 \%$ of $\Delta$ gerKA-KC spores germinated with $\mathrm{BHI}, \sim 90 \%$ did so when incubated with sheep blood serum, indicating that serum germination factor significantly increased germination of $\triangle$ gerKA-KC spores. The implications of these findings are that spores with defective GRs are still able to germinate inside the host, and that endogenous serum germination factor might be promoting in vivo germination. However, it is not yet clear how $C$. perfringens spores germinate in vivo without heat activation $\left(80^{\circ} \mathrm{C}, 10 \mathrm{~min}\right)$, a process known to increase the commitment of spores to germinate possibly through a GR-dependent pathway (Stewart et al., 1981; Yi \& Setlow, 2010; Zhang et al., 2010).

A second major conclusion offered by this work is that the host serum germination factor is likely to be serum lysozyme that triggers $C$. perfringens spore germination by directly degrading the spore peptidoglycan cortex. Indeed, the fact that C. perfringens spores with defects in the major components of the cortex lytic machinery were able to significantly germinate and show increased c.f.u. counts when incubated with serum, suggests that the serum germination factor has peptidoglycan hydrolysing activity. To our knowledge, the only host enzyme with peptidoglycan hydrolysing activity identified to date is lysozyme (Hankiewicz \& Swierczek, 1974; Pier et al., 2004; Prager \& Jolles, 1996; Reitamo et al., 1978), which hydrolyses the bacterial cell wall through cleavage of the linkage bond of $\mathrm{N}$-acetylmuramic acid and $\mathrm{N}$-acetyl-D-glucosamine residues of the peptidoglycan (Cole \& Ganz, 2005). Our Western blot analyses using monoclonal anti-human lysozyme antibody detected lysozyme-specific immunoreactive bands in HS and HMM fractions, but not in the LMM fraction, results that are consistent with the peptidoglycan hydrolysis activity observed in HS and HMM fractions. Our finding, that the serum germination factor is indeed lysozyme, was consistent with results from some early studies where purified lysozyme triggered germination of heat-injured and coat-deficient $C$. perfringens spores (Cassier \& Sebald, 1969; Cassier \& Ryter, 1971; Sacks, 1981). However, our current study advanced our understanding of the mechanism of lysozyme-triggered spore germination by showing that serum lysozyme triggered germination of $C$. perfringens spores by bypassing the GRs and directly degrading the spore peptidoglycan cortex.

A third and perhaps the most important conclusion from this work is that the host's lysozyme forms a high molecular mass complex (HMMC) in the serum, as a lysozyme-specific band of $\sim 120 \mathrm{kDa}$, but not a free lysozyme-specific band of $\sim 16 \mathrm{kDa}$, was detected only in HMM fractions of serum. It is possible that the lysozyme-like proteins (of molecular masses of $14-16 \mathrm{kDa}$ ) might be interacting with more abundant protein species present in the serum, forming a HMMC incapable of passing through the $30 \mathrm{kDa}$ cut-off filter. Although a previous study (Zhou et al., 2004) successfully fractionated a LMM fraction $(<30 \mathrm{kDa})$ from HS using a YM-30 Centricon ultrafiltration device, the sample was previously boiled, followed by other treatments, in contrast to the native conditions used in this study. An obvious question is whether this HMMC would enhance the ability of lysozyme to interact with pathogenic bacteria. Indeed, while the majority of untreated $\Delta c s p B$ spores became phase dark (indicating full spore core hydration) when incubated with HS, the majority of untreated $\Delta c s p B$ spores became phase grey (indicating partial spore core hydration) 
when treated with lysozyme. In contrast, serum and purified lysozyme were equally efficient in decoated $\Delta c s p B$ spores. In this context, although there are many other components in the serum that might enhance spore germination triggered by serum lysozyme, it might be possible that the serum HMMC might facilitate spore-lysozyme interaction in the presence of spore coats and also perhaps increase lysozyme's peptidoglycan hydrolysing activity. However, further studies will be needed to prove this.

\section{ACKNOWLEDGEMENTS}

This work was supported by a grant from the Agricultural Research Foundation of Oregon State University and by a Department of Defense Multi-disciplinary University Research Initiative (MURI) award through the US Army Research Laboratory and the US Army Research Office under contract number W911NF-09-1-0286 (all to M.R.S.).

\section{REFERENCES}

Amimoto, K., Noro, T., Oishi, E. \& Shimizu, M. (2007). A novel toxin homologous to large clostridial cytotoxins found in culture supernatant of Clostridium perfringens type C. Microbiology 153, 1198-1206.

Awad, M. M., Ellemor, D. M., Boyd, R. L., Emmins, J. J. \& Rood, J. I. (2001). Synergistic effects of alpha-toxin and perfringolysin $\mathrm{O}$ in Clostridium perfringens-mediated gas gangrene. Infect Immun 69, 7904-7910.

Cassier, M. \& Ryter, A. (1971). [A Clostridium perfringens mutant producing coatless spores by lysozyme-dependent germination]. Ann Inst Pasteur (Paris) 121, 717-732 (in French).

Cassier, M. \& Sebald, M. (1969). [Lysozyme-dependent germination of spores of Clostridium perfringens ATCC 3624 after heat treatment]. Ann Inst Pasteur (Paris) 117, 312-324 (in French).

Cole, A. M. \& Ganz, T. (2005). Defensins and other antimicrobial peptides: innate defense of mucosal surfaces. In Colonization of Mucosal Surfaces, pp. 17-34. Edited by J. P. Nataro, P. S. Cohen, H. L. T. Mobley \& J. N. Weiser. Washington, DC: American Society for Microbiology.

Duncan, C. L. \& Strong, D. H. (1968). Improved medium for sporulation of Clostridium perfringens. Appl Microbiol 16, 82-89.

Giebel, J. D., Carr, K. A., Anderson, E. C. \& Hanna, P. C. (2009). The germination-specific lytic enzymes SleB, CwlJ1, and CwlJ2 each contribute to Bacillus anthracis spore germination and virulence. J Bacteriol 191, 5569-5576.

Hankiewicz, J. \& Swierczek, E. (1974). Lysozyme in human body fluids. Clin Chim Acta 57, 205-209.

Huang, I. H., Waters, M., Grau, R. R. \& Sarker, M. R. (2004). Disruption of the gene (spo0A) encoding sporulation transcription factor blocks endospore formation and enterotoxin production in enterotoxigenic Clostridium perfringens type A. FEMS Microbiol Lett 233, 233-240.

Inoue, K., Tanigawa, Y., Takubo, M., Satani, M. \& Amano, T. (1959). Quantitative studies on immune bacteriolysis. II. The role of lysozyme in immune bacteriolysin. Biken's J 2, 1-20.

Keyburn, A. L., Boyce, J. D., Vaz, P., Bannam, T. L., Ford, M. E., Parker, D., Di Rubbo, A., Rood, J. I. \& Moore, R. J. (2008). NetB, a new toxin that is associated with avian necrotic enteritis caused by Clostridium perfringens. PLoS Pathog 4, e26.
Klobutcher, L. A., Ragkousi, K. \& Setlow, P. (2006). The Bacillus subtilis spore coat provides "eat resistance" during phagocytic predation by the protozoan Tetrahymena thermophila. Proc Natl Acad Sci U S A 103, 165-170.

Kokai-Kun, J. F., Songer, J. G., Czeczulin, J. R., Chen, F. \& McClane, B. A. (1994). Comparison of Western immunoblots and gene detection assays for identification of potentially enterotoxigenic isolates of Clostridium perfringens. J Clin Microbiol 32, 2533-2539.

Laaberki, M. H. \& Dworkin, J. (2008). Role of spore coat proteins in the resistance of Bacillus subtilis spores to Caenorhabditis elegans predation. J Bacteriol 190, 6197-6203.

Lund, B. M. \& Peck, M. W. (1994). Heat resistance and recovery of spores of non-proteolytic Clostridium botulinum in relation to refrigerated, processed foods with an extended shelf-life. Soc Appl Bacteriol Symp Ser 23, 115S-128S.

McClane, B. A. (2007). Clostridium perfringens. In Food Microbiology: Fundamentals and Frontiers, pp. 423-444. Edited by M. P. Doyle \& L. R. Beuchat. Washington, DC: American Society for Microbiology.

Michael, J. G. \& Braun, W. (1964). Analysis of sequential stages in serum bactericidal reactions. J Bacteriol 87, 1067-1072.

Paidhungat, M., Ragkousi, K. \& Setlow, P. (2001). Genetic requirements for induction of germination of spores of Bacillus subtilis by $\mathrm{Ca}^{2+}$-dipicolinate. J Bacteriol 183, 4886-4893.

Paredes-Sabja, D. \& Sarker, M. R. (2009). Clostridium perfringens sporulation and its relevance to pathogenesis. Future Microbiol 4, 519-525.

Paredes-Sabja, D., Setlow, B., Setlow, P. \& Sarker, M. R. (2008a). Characterization of Clostridium perfringens spores that lack SpoVA proteins and dipicolinic acid. J Bacteriol 190, 4648-4659.

Paredes-Sabja, D., Torres, J. A., Setlow, P. \& Sarker, M. R. (2008b). Clostridium perfringens spore germination: characterization of germinants and their receptors. J Bacteriol 190, 1190-1201.

Paredes-Sabja, D., Setlow, P. \& Sarker, M. R. (2009a). Role of GerKB in germination and outgrowth of Clostridium perfringens spores. Appl Environ Microbiol 75, 3813-3817.

Paredes-Sabja, D., Setlow, P. \& Sarker, M. R. (2009b). The protease CspB is essential for initiation of cortex hydrolysis and dipicolinic acid (DPA) release during germination of Clostridium perfringens type A food poisoning isolates. Microbiology 155, 3464-3472.

Paredes-Sabja, D., Setlow, P. \& Sarker, M. R. (2009c). SleC is essential for cortex peptidoglycan hydrolysis during germination of spores of the pathogenic bacterium Clostridium perfringens. J Bacteriol 191, 2711-2720.

Paredes-Sabja, D., Setlow, P. \& Sarker, M. R. (2011). Germination of spores of Bacillales and Clostridiales species: mechanisms and proteins involved. Trends Microbiol 19, 85-94.

Peck, M. W., Fairbairn, D. A. \& Lund, B. M. (1992). Factors affecting growth from heat-treated spores of non-proteolytic Clostridium botulinum. Lett Appl Microbiol 15, 152-155.

Pier, G. B., Lyczak, J. B. \& Wetzler, L. M. (2004). Immunology, Infection, and Immunity. Washington, DC: American Society for Microbiology.

Prager, E. M. \& Jolles, P. (1996). Lysozyme: a model enzyme in protein chemistry. In Lysozymes: Model Enzymes in Biochemistry and Biology, pp. 9-87. Edited by P. Jolles. Basel: Birkhauser-Verlag.

Reitamo, S., Klockars, M., Adinolfi, M. \& Osserman, E. F. (1978). Human lysozyme (origin and distribution in health and disease). Ric Clin Lab 8, 211-231.

Sacks, L. E. (1981). Influence of cations on lysozyme-induced germination of coatless spores of Clostridium perfringens 8-6. Biochim Biophys Acta 674, 118-127.

Sarker, M. R., Shivers, R. P., Sparks, S. G., Juneja, V. K. \& McClane, B. A. (2000). Comparative experiments to examine the effects of 
heating on vegetative cells and spores of Clostridium perfringens isolates carrying plasmid genes versus chromosomal enterotoxin genes. Appl Environ Microbiol 66, 3234-3240.

Setlow, P. (2003). Spore germination. Curr Opin Microbiol 6, 550556.

Shimizu, T., Ohtani, K., Hirakawa, H., Ohshima, K., Yamashita, A., Shiba, T., Ogasawara, N., Hattori, M., Kuhara, S. \& Hayashi, H. (2002). Complete genome sequence of Clostridium perfringens, an anaerobic flesh-eater. Proc Natl Acad Sci U S A 99, 996-1001.

Stewart, G. S., Johnstone, K., Hagelberg, E. \& Ellar, D. J. (1981). Commitment of bacterial spores to germinate. A measure of the trigger reaction. Biochem J 198, 101-106.
Yi, X. \& Setlow, P. (2010). Studies of the commitment step in the germination of spores of Bacillus species. J Bacteriol 192, 3424-3433.

Zhang, P., Garner, W., Yi, X., Yu, J., Li, Y. Q. \& Setlow, P. (2010). Factors affecting variability in time between addition of nutrient germinants and rapid dipicolinic acid release during germination of spores of Bacillus species. J Bacteriol 192, 3608-3619.

Zhao, Y. \& Melville, S. B. (1998). Identification and characterization of sporulation-dependent promoters upstream of the enterotoxin gene (cpe) of Clostridium perfringens. J Bacteriol 180, 136-142.

Zhou, M., Lucas, D. A., Chan, K. C., Issaq, H. J., Petricoin, E. F., III, Liotta, L. A., Veenstra, T. D. \& Conrads, T. P. (2004). An investigation into the human serum "interactome". Electrophoresis 25, 1289-1298. 